\title{
Epigenetic landscapes of intracranial aneurysm risk haplotypes implicate enhancer function of endothelial cells and fibroblasts in dysregulated gene expression
}

Kerry E. Poppenberg ${ }^{1,2}$, Haley R. Zebraski 1,3, Naval Avasthi ${ }^{1,3}$, Muhammad Waqas ${ }^{1,2}$, Adnan H. Siddiqui ${ }^{1,2}$, James N. Jarvis ${ }^{4}$ and Vincent M. Tutino $1,2,3,5,6^{*}$

\begin{abstract}
Background: Genome-wide association studies have identified many single nucleotide polymorphisms (SNPs) associated with increased risk for intracranial aneurysm (IA). However, how such variants affect gene expression within IA is poorly understood. We used publicly-available ChIP-Seq data to study chromatin landscapes surrounding risk loci to determine whether IA-associated SNPs affect functional elements that regulate gene expression in cell types comprising IA tissue.
\end{abstract}

Methods: We mapped 16 significant IA-associated SNPs to linkage disequilibrium (LD) blocks within human genome. Using ChIP-Seq data, we examined these regions for presence of H3K4me1, H3K27ac, and H3K9ac histone marks (typically associated with latent/active enhancers). This analysis was conducted in several cell types that are present in IA tissue (endothelial cells, smooth muscle cells, fibroblasts, macrophages, monocytes, neutrophils, T cells, B cells, NK cells). In cell types with significant histone enrichment, we used HiC data to investigate topologically associated domains (TADs) encompassing the LD blocks to identify genes that may be affected by IA-associated variants. Bioinformatics were performed to determine the biological significance of these genes. Genes within HiC-defined TADs were also compared to differentially expressed genes from RNA-seq/microarray studies of IA tissues.

Results: We found that endothelial cells and fibroblasts, rather than smooth muscle or immune cells, have significant enrichment for enhancer marks on IA risk haplotypes $(p<0.05)$. Bioinformatics demonstrated that genes within TADs subsuming these regions are associated with structural extracellular matrix components and enzymatic activity. The majority of histone marked TADs (83\% fibroblasts [IMR90], 77\% HUVEC) encompassed at least one differentially expressed gene from IA tissue studies.

Conclusions: These findings provide evidence that genetic variants associated with IA risk act on endothelial cells and fibroblasts. There is strong circumstantial evidence that this may be mediated through altered enhancer function, as genes in TADs encompassing enhancer marks have also been shown to be differentially expressed in IA tissue. These genes are largely related to organization and regulation of the extracellular matrix. This study builds upon our

\footnotetext{
*Correspondence: vincentt@buffalo.edu

${ }^{1}$ Canon Stroke and Vascular Research Center, University at Buffalo, Clinical

and Translational Research Center, 875 Ellicott Street, Buffalo, NY 14214,

USA

Full list of author information is available at the end of the article
}

(C) The Author(s) 2021. Open Access This article is licensed under a Creative Commons Attribution 4.0 International License, which permits use, sharing, adaptation, distribution and reproduction in any medium or format, as long as you give appropriate credit to the original author(s) and the source, provide a link to the Creative Commons licence, and indicate if changes were made. The images or other third party material in this article are included in the article's Creative Commons licence, unless indicated otherwise in a credit line to the material. If material is not included in the article's Creative Commons licence and your intended use is not permitted by statutory regulation or exceeds the permitted use, you will need to obtain permission directly from the copyright holder. To view a copy of this licence, visit http://creativecommons.org/licenses/by/4.0/. The Creative Commons Public Domain Dedication waiver (http://creativeco mmons.org/publicdomain/zero/1.0/) applies to the data made available in this article, unless otherwise stated in a credit line to the data. 
previous (Poppenberg et al., BMC Med Genomics, 2019) by including a more diverse set of data from additional cell types and by identifying potential affected genes (i.e. those in TADs).

Keywords: Intracranial aneurysm, Epigenetics, Genetic risk, Histone mark, Topologically associated domain

\section{Background}

Intracranial aneurysms (IAs) are focal dilations of the cerebral vessels that are present in approximately $2-6 \%$ of the general population [1]. IA is a complex disease trait, in which heritable genetic variants are known to play an important role [2]. Indeed, family history of IA has been shown to be a significant risk factor for developing an aneurysm (odds ratio-OR 4) [3]. In studying genetic risk for IA, genome-wide association studies (GWAS) have identified many aneurysm-associated single nucleotide polymorphisms (SNPs) [4-12], the most significant existing at 2q32.1 (PLCL1) [12], 8q11.23-q12.1 (SOX17) [12], 9p21.3 (CDKN2A-CDKN2B) [12], 18q11.2 (RBBP8) [10], 13q13.1 (STARD13) [10], and 10q24.32.12 [10]. Notably, many IA-risk loci include functional, noncoding regions, suggesting that genetic risk may operate on these regulatory elements, including enhancers [13], that influence gene expression, rather than on the structure of the gene product itself [14]. However, deriving mechanistic insights from GWAS data alone is problematic, since the true variant(s) that confer risk are not usually known. SNPs used to tag risk loci on GWAS are typically in linkage disequilibrium (LD) with dozens or hundreds (sometimes even thousands) of other SNPs, most of which have no influence on disease risk [15-17]. One way to gain insight into mechanisms through which genetic variants associated with IA exert risk is to examine the chromatin structures across the entire haplotypes.

In a recent study by our group [18], we explored the chromatin landscapes associated with the haplotypes encompassing 16 SNPs strongly associated with IA that were reported in a meta-analysis by Alg et al. [19]. Using ChIP-seq data available from the ENCODE project, we queried the LD blocks that incorporated the relevant tag SNPs in endothelial cells (ECs), monocytes, neutrophils, and peripheral blood mononuclear cells (PBMCs) to determine whether there was epigenetic evidence that genetic risk was conferred more predominantly in circulating immune cells or the vascular endothelium [18]. From this data, we found that the cells of the endothelium, rather than immune cell types, were statistically significantly enriched (compared to genome background) for H3K4meland H3K27ac histone marks, epigenetic features usually associated with functional enhancers. Enhancers, noncoding regulatory elements that play an important role in modulating gene expression, serve as regulators that fine-tune gene expression to fit specific physiologic contexts [20]. Thus, our data suggest that known genetic risk factors for IA may alter gene expression in the vessel wall through modulation of enhancer activity. Yet, it is known that the presence of H3K4me1 and H3K27ac at specific genomic locations is not sufficient to establish that the region in question truly has enhancer activity and is thus involved in active gene transcription. The locations of the marks are also not sufficient to determine the specific genes that might be affected, as it is widely known that enhancers can regulate more than one gene, and may not regulate the gene(s) in closest proximity.

In this study, we sought to gain further insight into how IA risk loci impinge on noncoding, regulatory elements and influence gene expression by interrogating additional chromatin features that encompass the established IA risk loci. To do this, we took advantage of the fact that chromatin is organized in distinct DNA looping structures, or topologically associated domains (TADs), that are demarcated by the presence of the anchoring structures CTCF and cohesion. As Gasperini et al. detailed, enhancers do not always influence the nearest gene in terms of linear genomic distance, but almost invariably regulate genes within the same TAD [21]. Seeing as our previous analysis demonstrated that the vascular tissue may confer genetic risk for IA, we examined histone marks and their associated TADs that subsume IA-associated LD blocks in cells present in IA tissue (specifically ECs [22], B cells [23], fibroblasts [22], macrophages and monocytes [22, 23], natural killer (NK) cells [24], neutrophils [22, 25], smooth muscle cells (SMCs) [22, 26], and $\mathrm{T}$ cells $[22,23])$. After assessing histone marks in each cell type, we investigated likely enhancer targets by querying the overlap with TAD data derived from $\mathrm{HiC}$ experiments [27] and performing bioinformatics analyses on genes found within the TADs. If genetic variants are operating on genes in these TADs, we might expect them to be differentially expressed in the IA tissue. To test this idea, we compared identified target genes within HiC-defined TADs with differentially expressed genes reported from RNA sequencing or gene chip microarray studies performed on human IA tissue.

\section{Methods \\ Identification of LD blocks}

We analyzed 16 of 19 IA-associated SNPs identified in a meta-analysis by Alg et al. [19] to have been reported as 
significantly associated with IA in two or more previous publications. We note that SNPs identified on GWAS do not identify the causal polymorphism, rather, they index a larger genetic region where risk may operate. Therefore, the causal SNP may be anywhere within the LD block. For this reason, we examined the haplotype blocks (or LD blocks) surrounding the index SNPs of interest, as these regions are inherited together, with an r-squared of 0.9 . We used the UCSC LiftOver tool (https://genome.ucsc. edu/cgi-bin/hgLiftOver) to identify the LD block positions in hg38 for use in our histone enrichment analysis.

\section{Identification of H3K4me1/H3K27ac/H3K9ac histone marks within LD blocks}

We used the Cistrome data browser to find hg38 ChIPSeq peak data for H3K27ac, H3K4me1, and H3K9ac histone marks $[28,29]$. All data from Cistrome is processed using ChiLin pipeline [30], which uses BWA to align raw data and MACS2 to call peaks. Cistrome also reports 7 quality control metrics for each dataset, including sequence quality and ChIP enrichment. We specifically investigated cell types found in the aneurysm walls, as reported by histological studies of human IA tissues [23, 31-33]. These included human B lymphocytes, ECs (aortic, HAEC, and umbilical vein, HUVEC), fibroblasts (skin and lung), macrophages, monocytes, NK cells, neutrophils, SMCs, and T lymphocytes (CD4+ and CD8+). For each, we selected peaks files from the ENCODE consortium or those with high quality scores. Additional file 1: Table S1 reports the data source and GEO or ENCODE accession number for each of the data files used. Accession numbers also listed in availability of data and materials section. To identify if any of the histone peaks overlapped with our LD blocks of interest, we used the intersect command within the BEDTools command line software.

To test for significance enrichment of the marks, we empirically calculated the $p$ value using the $\mathrm{z}$-score from a histogram based on 1000 iterations. In brief, we first created 16 random regions from hg38 genome file with an average length equal to the average length of the IAassociated LD blocks. We then used the intersect command to determine how many of the 16 random regions overlapped with the peaks for the various cell types' histone marks. A histogram was created to depict the distribution over all 1000 iterations and to calculate the associated mean $(\mu)$ and standard deviation $(\sigma)$. We calculated the $\mathrm{z}$-score using the following equation: $z=\frac{x-\mu}{\sigma}$ in which $\mathrm{x}$ is the number of intersections between the IA-associated LD blocks and the peak file of interest. The $p$ value was then determined from the $\mathrm{z}$-score; $p$ value $<0.05$ was deemed significant. For cell types with significant H3K27ac marks, we also queried H3K9me3 marks, which indicate that chromatin is closed and inaccessible.

\section{Identification of TADs}

To identify TADs encompassing the IA-associated risk haplotypes, as well as the genes within these loop structures, we mined existing $\mathrm{HiC}$ data available on the 3D Genome browser [34]. $\mathrm{HiC}$ is a non-directed technique that allows one to discern pairwise contacts between any two regions in the genome based on their physical proximity [35]. For cell types in which there were significant association with enhancer marks, all genes encompassed within the TADs subsuming the regions with significant enhancer marks were recorded and used in bioinformatics analyses.

\section{Bioinformatics}

For genes identified within the TADs that encompass the IA risk haplotypes in which there were significant association with H3K9ac/H3K4me1/H3K27ac marks, we performed gene ontology enrichment $(\mathrm{GO})$ analysis via g:Profiler under the default settings. GO terms were reported if the input gene list was enriched for any term to a greater degree than what would be expected by chance (adjusted $p$ value $<0.05$ ) [36]. The genes within the relevant TADs were also analyzed using Ingenuity Pathway Analysis (IPA) [37]. Here, each gene identifier was mapped to its corresponding gene object in the Ingenuity Knowledge Base and overlaid onto a molecular network derived from information accumulated in the Knowledge Base. Related disease or function annotations with a Benjamini-Hochberg $p$ value $<0.01$ that were identified using at least 3 of the input genes were deemed significant. Gene networks were algorithmically generated based on their "connectivity" derived from known interactions between the products of the input genes. Networks were considered significant if their $\mathrm{p}$-scores were $>21$.

\section{Comparison with IA tissue differential expression studies}

In order to gather further evidence that enhancers on the risk haplotypes influence gene expression in the cell types of interest, we compared the genes from IA-associated TADs with differentially expressed genes from IA tissue studies. The rationale for this approach is the strong experimental evidence that enhancers almost invariably regulate genes within the same TAD [21]. Thus, we sought to determine whether there were any likely candidate genes whose expression levels might be impacted by genetic variants within the H3K4me1/H3K27ac/ H3K9ac-marked regions. We included 8 studies that investigated differential expression between aneurysm and control tissues or between ruptured IAs and unruptured IAs. Differentially expressed genes between IA and 
control tissues were reported by Aoki [38], Kleinloog [39], Li [40], Pera [41], Shi [42], and Wang [43]. Differential expression between ruptured and unruptured IAs was investigated by Kleinloog [39], Kurki [44], Nakaoka [45], and Pera [41]. We compared their published lists of differentially expressed genes (DEGs) with those found in IA-associated TADs.

\section{Results}

\section{Queried LD blocks}

In this study, we analyzed the epigenetic landscapes of 16 LD blocks, which are reported in Table 1 (Additional file 1: Table S2 also reports both hg19 and hg38 positions for the LD blocks, as they were originally identified in hg19 but mapped to hg38 to agree with Cistrome data). Based on our analysis, we found that the majority $(12 / 16)$ of the sentinel SNPs were located within noncoding regions, as is common in complex traits. The remaining 4 index SNPs were found to be within exons, although it cannot be assumed that the SNP impacts the coding function of the given gene [46].

\section{Histone marks for enhancers are significantly enriched in endothelial cells and fibroblasts}

We investigated epigenetic features associated with enhancer function in the LD blocks for a variety of cell types known to be present in the aneurysmal tissue (B cells, ECs, fibroblasts, macrophages, monocytes, NK cells, neutrophils, SMCs, T cells) using data from the Cistrome browser. HUVECs demonstrated significant enrichment for histone marks in IA-associated LD blocks as compared to the randomly-generated genomic regions (H3K4me1 $p=0.00025$, H3K9ac $p=0.014$, H3K27ac $p=0.0031$, consistent with our previously published results [18]. As shown in Table 2, 7 of the 16 queried LD blocks had enrichment for all 3 histone marks in HUVEC data. While only H3K27ac data was available for aortic ECs, ChIP-seq peaks were present in 8 regions, which was significant $(p=0.011)$. Skin fibroblasts also demonstrated significant enhancer mark enrichment (H3K4me1 $p=0.0091$, H3K9ac $p=0.010$, H3K27ac $p=0.0027$ ) and had all 3 marks in 5 LD blocks. NK cells and T lymphocytes had the fewest H3K4me1/H3K27ac/H3K9ac peaks of the cell types examined.

We examined H3K9me3 marks in those cell types with significant marks, namely HUVECs, lung fibroblasts, and skin fibroblasts (accession numbers provided in availability of data and materials section and Additional file 1: Table S1). While HAEC had significant H3K27ac marks, there was no H3K9me3 dataset of sufficient quality to examine. None of the haplotypes queried encompassed H3K9me3 marks.

For example, we show the landscape around a large LD block associated with the intergenic SNP rs1429412, which is nearest to ANKRD44, in Fig. 1A. This region was particularly rich containing all marks for all cell types except for H3K4me1 in SMCs. Another example containing $\mathrm{H} 3 \mathrm{~K} 4 \mathrm{me} 1 / \mathrm{H} 3 \mathrm{~K} 27 \mathrm{ac} / \mathrm{H} 3 \mathrm{~K} 9 \mathrm{ac}$ peaks in ECs and fibroblasts was centered around rs1800796, which falls within the first intron of IL6, an important

Table 1 IA risk associated SNPs and the associated linkage disequilibrium blocks

\begin{tabular}{lllll}
\hline Sentinel SNP & SNP Location & Nearest Gene & LD Block (hg38) & Length (bp) \\
\hline rs3767137 & Intronic & HSPG2 & chr1:21834230-21841817 & 7587 \\
rs1800255 & Exonic & COL3A1 & chr2:188976887-189003156 & chr2:197283467-197358397 \\
rs1429412 & Intergenic & ANKRD44 & chr2:197676674-197766990 & 74,930 \\
rs700651 & Intronic & BOLL & chr4:147444187-147493499 & 90,316 \\
rs6841581 & Intergenic & EDNRA & chr5:83509605-83530435 & 20,830 \\
rs251124 & Intronic & VCAN & chr7:15454259-15466904 & 12,645 \\
rs4628172 & Intronic & AGMO & chr7:22726627-22732119 & 5492 \\
rs1800796 & Intronic & IL6 & chr7:94413927-94420044 & 6117 \\
rs42524 & Exonic & COL1A2 & chr8:54397171-54415556 & 18,385 \\
rs10958409 & Intergenic & - & chr8:54509054-54549764 & 40,710 \\
rs9298506 & Intergenic & - & chr9:22072265-22125504 & 53,239 \\
rs2891168 & Intronic & CDKN2B-AS1 & chr9:22077086-22125504 & 48,418 \\
rs10757278 & Intergenic & CDKN2B-AS1 & chr12:95095355-95123067 & 27,712 \\
rs6538595 & Intronic & FGD6 & chr14:94612340-94614466 & 2126 \\
rs4934 & Exonic & SERPINA3 & chr20:17613385-17619469 & 6084 \\
rs1132274 & Exonic & RRBP1 & &
\end{tabular}

Bolded SNPs were chosen as examples in Fig. 1, Fig. 2, and Additional file 1: Fig. S1

hg, human genome; IA, intracranial aneurysm; rs, reference SNP cluster ID; SNP, single nucleotide polymorphism; chr, chromosome; LD, linkage disequilibrium 
Table 2 Histone marks present in IA risk associated linkage disequilibrium blocks

\begin{tabular}{|c|c|c|c|c|c|c|c|c|c|c|c|c|c|c|c|c|c|c|}
\hline $\begin{array}{l}\text { Cell } \\
\text { Type }\end{array}$ & $\begin{array}{l}\text { Histone } \\
\text { Mark }\end{array}$ & $\frac{\hat{n}}{\hat{\sigma}}$ & 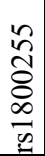 & 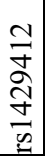 & $\begin{array}{l}\bar{n} \\
8 \\
8 \\
0 \\
0\end{array}$ & $\begin{array}{l}\bar{\infty} \\
\text { in } \\
\dot{\Phi} \\
\infty \\
0 \\
0\end{array}$ & 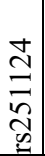 & 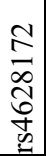 & 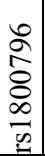 & 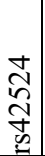 & 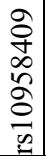 & 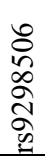 & 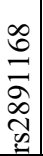 & 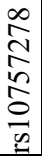 & $\begin{array}{l}n \\
\tilde{n} \\
\infty \\
n \\
\tilde{n} \\
b \\
\vdots \\
\vdots\end{array}$ & 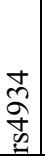 & 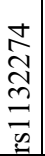 & p-val. \\
\hline HAEC & $\mathrm{H} 3 \mathrm{~K} 27 \mathrm{ac}$ & & $\mathrm{Y}$ & $\mathrm{Y}$ & $\mathrm{Y}$ & & $\mathrm{Y}$ & & $\mathrm{Y}$ & & & & $\mathrm{Y}$ & $\mathrm{Y}$ & $\mathrm{Y}$ & & & $0.0113 \dagger$ \\
\hline \multirow{3}{*}{ HUVEC } & H3K27ac & & $\mathrm{Y}$ & $\mathrm{Y}$ & $\mathrm{Y}$ & & & & $\bar{Y}$ & & $\bar{Y}$ & $\mathrm{Y}$ & $\overline{\mathrm{Y}}$ & $\mathrm{Y}$ & $\mathrm{Y}$ & & & $0.0031 \dagger$ \\
\hline & $\mathrm{H} 3 \mathrm{~K} 4 \mathrm{me} 1$ & & $\mathrm{Y}$ & $\mathrm{Y}$ & $\mathrm{Y}$ & $\mathrm{Y}$ & $\mathrm{Y}$ & Y & Y & Y & Y & $\mathrm{Y}$ & Y & $\mathrm{Y}$ & $\mathrm{Y}$ & & & $0.0003 \dagger$ \\
\hline & H3K9ac & & $\mathrm{Y}$ & $\mathrm{Y}$ & $\mathrm{Y}$ & & & & $\mathrm{Y}$ & & & & $\mathrm{Y}$ & $\mathrm{Y}$ & $\mathrm{Y}$ & & & $0.0142 \dagger$ \\
\hline \multirow[b]{2}{*}{ SMC } & H3K27ac & & $\mathrm{Y}$ & $\mathrm{Y}$ & & & & & $\mathrm{Y}$ & & & & $\mathrm{Y}$ & $\mathrm{Y}$ & & & & 0.2685 \\
\hline & $\begin{array}{c}\text { H3K4me1 } \\
\text { H3K9ac }\end{array}$ & & $\mathrm{Y}$ & $\mathrm{Y}$ & $\mathrm{Y}$ & & & & Y & & $\mathrm{Y}$ & & Y & Y & & & & $\begin{array}{l}0.8192 \\
0.0542\end{array}$ \\
\hline \multirow{2}{*}{$\begin{array}{r}\text { Fib. } \\
\text { (Lung) }\end{array}$} & H3K27ac & & $\mathrm{Y}$ & $\mathrm{Y}$ & $\mathrm{Y}$ & & $\mathrm{Y}$ & & $\mathrm{Y}$ & & & & $\mathrm{Y}$ & $\mathrm{Y}$ & $\mathrm{Y}$ & & & $0.0079 \dagger$ \\
\hline & $\begin{array}{c}\text { H3K4me1 } \\
\text { H3K9ac }\end{array}$ & & & $\begin{array}{l}\mathrm{Y} \\
\mathrm{Y}\end{array}$ & $\mathrm{Y}$ & $\begin{array}{l}\mathrm{Y} \\
\mathrm{Y}\end{array}$ & & & Y & & & & $\mathrm{Y}$ & $\mathrm{Y}$ & $\mathrm{Y}$ & & $\mathrm{Y}$ & $\begin{array}{l}0.0813 \\
0.1224\end{array}$ \\
\hline \multirow{3}{*}{$\begin{array}{r}\text { Fib. } \\
\text { (Skin) }\end{array}$} & $\mathrm{H} 3 \mathrm{~K} 27 \mathrm{ac}$ & & $\mathrm{Y}$ & $\mathrm{Y}$ & $\mathrm{Y}$ & & & $\mathrm{Y}$ & $\mathrm{Y}$ & $\bar{Y}$ & & & $\bar{Y}$ & $\bar{Y}$ & $\mathrm{Y}$ & & & $0.0027 \dagger$ \\
\hline & $\mathrm{H} 3 \mathrm{~K} 4 \mathrm{me} 1$ & & & Y & $\mathrm{Y}$ & $\mathrm{Y}$ & & & & & & & $\mathrm{Y}$ & Y & $\mathrm{Y}$ & & Y & $0.0091 \dagger$ \\
\hline & H3K9ac & & & $\mathrm{Y}$ & $\mathrm{Y}$ & $\mathrm{Y}$ & & & & & & & $\mathrm{Y}$ & $\mathrm{Y}$ & $\mathrm{Y}$ & & & $0.0103 \dagger$ \\
\hline \multirow{2}{*}{ M0 } & H3K27ac & & & $\mathrm{Y}$ & $\mathrm{Y}$ & & & & & & & & $\mathrm{Y}$ & $\mathrm{Y}$ & $\mathrm{Y}$ & & & 0.1655 \\
\hline & $\mathrm{H} 3 \mathrm{~K} 4 \mathrm{me} 1$ & & & Y & $\mathrm{Y}$ & & & & & & & & Y & $\mathrm{Y}$ & $\mathrm{Y}$ & & & 0.0727 \\
\hline \multirow{3}{*}{ Mono. } & H3K27ac & & & $\bar{Y}$ & $\mathrm{Y}$ & & $\mathrm{Y}$ & & & & & & & & $\mathrm{Y}$ & & & 0.4719 \\
\hline & H3K4me1 & & & Y & $\mathrm{Y}$ & & Y & & & & & & Y & $\mathrm{Y}$ & $\mathrm{Y}$ & & Y & 0.2212 \\
\hline & H3K9ac & & & Y & $\mathrm{Y}$ & & & & & & & & & & $\mathrm{Y}$ & & Y & 0.1847 \\
\hline \multirow{2}{*}{ PNL } & H3K27ac & & & $\mathrm{Y}$ & $\mathrm{Y}$ & & & & & & & & & & & & $\mathrm{Y}$ & 0.6953 \\
\hline & $\mathrm{H} 3 \mathrm{~K} 4 \mathrm{me} 1$ & & & Y & $\mathrm{Y}$ & & & & & & & & & & $\mathrm{Y}$ & & Y & 0.6841 \\
\hline \multirow{3}{*}{ B Lym. } & $\mathrm{H} 3 \mathrm{~K} 27 \mathrm{ac}$ & & & Y & $\mathrm{Y}$ & & & & & & & & & & & & & 0.7688 \\
\hline & $\mathrm{H} 3 \mathrm{~K} 4 \mathrm{me} 1$ & & $\mathrm{Y}$ & $\mathrm{Y}$ & $\mathrm{Y}$ & $\mathrm{Y}$ & Y & & & & & & $\mathrm{Y}$ & $\mathrm{Y}$ & Y & & & 0.1065 \\
\hline & H3K9ac & & & $\mathrm{Y}$ & $\mathrm{Y}$ & & & & & & & & & & & & & 0.6135 \\
\hline \multirow{3}{*}{$\begin{array}{l}\text { T Lym. } \\
\text { (CD4+) }\end{array}$} & H3K27ac & & & $\mathrm{Y}$ & $\mathrm{Y}$ & & & & & & & & & & & & & 0.3476 \\
\hline & H3K4me1 & & & Y & $\mathrm{Y}$ & & & & Y & & & & $\mathrm{Y}$ & $\mathrm{Y}$ & $\mathrm{Y}$ & & & 0.6256 \\
\hline & H3K9ac & & & $\mathrm{Y}$ & & & & & & & & & & & & & & 0.4022 \\
\hline \multirow{3}{*}{$\begin{array}{l}\text { T Lym. } \\
(\mathrm{CD} 8+)\end{array}$} & H3K27ac & & & $\mathrm{Y}$ & $\mathrm{Y}$ & & & & & & & & & & & & & 0.6990 \\
\hline & $\mathrm{H} 3 \mathrm{~K} 4 \mathrm{me} 1$ & & & $\mathrm{Y}$ & $\mathrm{Y}$ & & & & Y & & & & $\mathrm{Y}$ & $\mathrm{Y}$ & & & & 0.5421 \\
\hline & H3K9ac & & & $\mathrm{Y}$ & & & & & & & & & & & & & & 0.5548 \\
\hline \multirow{2}{*}{ NK } & H3K27ac & & & $\mathrm{Y}$ & $\mathrm{Y}$ & & & & & & & & & & & & $\mathrm{Y}$ & 0.3792 \\
\hline & $\mathrm{H} 3 \mathrm{~K} 4 \mathrm{me} 1$ & & & $\mathrm{Y}$ & $\mathrm{Y}$ & & & & & & & & & & & & & 0.8049 \\
\hline
\end{tabular}

The color of each cell indicates number of peaks in each region with enrichment (grey $=$ none, yellow $=1-5$, orange $=6-10$, red $=11$ or more) " $t$ " denotes significance

Fib., fibroblast; HAEC, human aortic endothelial cell; HUVEC, human umbilical vein endothelial cell; IA, intracranial aneurysm; lym., lymphocyte; M0, macrophage; mono., monocyte; NK, natural killer cell; p-val., p-value; PNL, polymorphonuclear leukocyte; rs, reference SNP cluster ID; SMC, smooth muscle cell; Y, yes

inflammatory cytokine that has been strongly implicated in IA pathogenesis (Fig. 1B) [47]. H3K27ac marks were prominent in HAECs, HUVECs, fibroblasts (lung and skin), and SMCs. Both HUVECs and T cells (CD4+ and CD8 +) expressed H3K4me1 marks within this haplotype. H3K9ac marks within this LD block were found in HUVECs, fibroblasts (lung), and SMCs. In this LD block, HUVECs were the only cell type to express the 3 queried histone marks. A similar pattern of histone mark enrichment was also present in the epigenetic landscape around rs10757278, an intergenic region flanking CDKN2B-AS1 (Fig. 1C). H3K27ac marks were prominent in HAECs, HUVECs, fibroblasts (lung and skin), SMCs, and macrophages. HUVECs, fibroblasts, macrophages, monocytes, and lymphocyte populations had H3K4me1 marks present within this haplotype, while the H3K9ac marks were found only in HUVECs, skin fibroblasts, and SMCs. HUVECs and skin fibroblasts both expressed all 3 queried histone marks in this block. In Fig. 1D, we present the landscape surrounding an intronic SNP for
FGD6, rs6538595. All marks were present in HUVECs, skin fibroblasts, and monocytes, while H3K27ac and H3K4me1 marks were present in lung fibroblasts and macrophages. Neutrophils, B cells, and CD $4+\mathrm{T}$ cells contained H3K4me1 marks. Figure 1 provides visualization of data reported in Table 2 for four example regions (bolded SNPs in Table 1). Landscapes surrounding other SNPs can be visualized in UCSC Genome Browser by loading data from Cistrome.

\section{Genes in IA-associated TADs reflect ontologies related to extracellular matrix}

For the IA-associated LD blocks with histone marks present, we identified TADs and the genes encompassed within them. While TAD boundaries have been found to be conserved $[48,49]$, there is evidence suggesting TADs do vary among cell types $[27,50,51]$. Therefore, we examined HUVEC and fibroblast TADs independently using $\mathrm{HiC}$ data from Rao et al. [27]. Because $\mathrm{HiC}$ data were unavailable for skin fibroblasts, we used data 
(See figure on next page.)

Fig. 1 Landscape around four example IA-associated LD blocks showing enhancer marks in three cell types. HUVECs in blue, fibroblasts (lung) in green, SMCs in purple. IA-associated LD block in orange, index SNP is yellow line. " + " denotes significance. Relative height of ChIP-seq peaks all scaled from 0-10 in Genome Browser. A Genome browser screenshot showing the IA-associated LD block identified by the intergenic tap SNP rs1429412 near ANKRD44. All three marks (H3K27ac, H3K4me1, and H3K9ac) were present for both HUVECs and fibroblasts. B Genome browser screenshot showing the IA-associated LD block for the IL6 locus identified by the intronic tag SNP, rs1800796. All three marks are present for HUVECs. C Genome browser screenshot showing the IA-associated LD block identified by the intergenic tag SNP rs 10757278 near CDKN2B-AS1. All three marks were present for HUVECs. D Genome browser screenshot showing the IA-associated LD block for the FGD6 locus identified by intronic tag SNP, rs6538595. All three marks were present for HUVECs

from IMR90 cells (fetal lung fibroblasts). Figure 2 demonstrates the TADs that surround our four example SNPs, rs1429412 (A), rs1800796 (B), rs10757278 (C), and rs6538595 (D) in HUVECs. The TADs for these same four regions in fibroblasts (IMR90 cells) are presented in Additional file 1: Fig. S1. We found 164 transcripts within the TADs for HUVECs and 153 transcripts within the IMR90 TADs. See Additional file 1: Table S3 for genes encompassed within each TAD. Between the two cell types, 114 transcripts were common, and many were related to structural components, such as COL1A2, COL3A1, HAPLN1, VCAN, and VEZT. The g:Profiler showed that in HUVECs, 1 biological process GO term, 3 cellular component GO terms, and 7 molecular function GO terms were significantly enriched (see Additional file 1: Table S4). These annotations demonstrate enrichment of lipoxygenase pathway, fibrillar collagen trimer, and extracellular matrix structural constituent and peptidase regulator function. Fewer ontologies were associated with fibroblast (IMR90) TAD genes, with only 2 significant GO terms (S-methyl-5-thioadensoine phosphorylase activity and purine-nucleoside phosphorylase activity).

We used IPA to further characterize disease and biological functions and to investigate potential gene interaction networks based on genes found in the TADs with histone mark enrichment. For HUVECs, there were 13 significantly enriched diseases and bio-functions, including vascular lesion, Loeys-Dietz syndrome, and EhlersDanlos syndrome (the full list of significant terms is presented in Additional file 1: Table S5). Network analysis showed 2 significant networks with P-scores of 33 and 30, respectively, which were populated by connections around collagens and ERK1/2 genes (first network) and connections around ELAVL1 and TP53 (second network). Generally, these networks were related to connective tissue disorders and cellular assembly and organization. For TAD genes associated in fibroblasts (IMR90), IPA did not return any significant disease and biological function annotations. However, there were 3 significant networks with P-scores of 27, 25, and 25, which had connections emanating from hubs of NF-KB and IL6 (first network), from hubs of MAPK and ERK (second network), and from hubs of ESR2 and TGFB1 (third network). The top network associated with each cell type is presented in Fig. 3 (A- HUVECs, B- fibroblasts); the remaining networks are provided in Additional file 1: Fig. S2. Broadly, these networks were related to nucleic acid metabolism, dermatological diseases and conditions, and cellular growth and proliferation. See Additional file 1: Table S6 for additional details on the networks described above.

\section{Genes in IA-associated TADs overlap with those differentially expressed in IA tissue}

We compared the genes within the HUVEC and IMR90 TADs surrounding the IA SNPs of interest to genes differentially expressed in aneurysm tissue, reported in 8 distinct studies (see Additional file 1: Table S7 for information related to these publications). In HUVECs, 11/13 (85\%) IA-associated TADs enriched for H3K4me1/ H3K27ac/H3K9ac histone marks also contained a differentially expressed gene in IA tissue. Similarly, 10/12 (83\%) TADs encompassed a tissue DEG in IMR90 cells. (See Additional file 1: Table S3). We first analyzed the tissue studies that compared IA tissue to control tissue. The results are presented in Table 3. In HUVECs, we found 12 differentially expressed genes identified in IA tissue studies that were also present within TADs encompassing IA haplotypes, four of which were reported in multiple studies (COL1A2, COL3A1, COL5A2, VCAN). Using the list of fibroblast TAD genes, we found 11 genes that were also reported as differentially expressed between IA and control tissue. Three of these genes were reported in more than one of the aforementioned studies: COL1A2, $C O L 3 A 1$, and $V C A N$. Examining the differential expression due to IA rupture, we found 12 genes were within IA-associated TADs of HUVECs. Two genes, GULP1 and PLCL1, were found in multiple studies. Nine genes present in IA-associated TADs of IMR90 fibroblasts were reported to be differentially expressed between unruptured and ruptured IA samples. Again, GULP1 and PLCL1 were present in multiple studies, with the addition of BFSP1. Combining results for IA vs. control tissue and ruptured vs. unruptured IA tissue, a total of $12 \%$ of transcripts within both HUVEC and IMR90 fibroblast 
A rs1429412

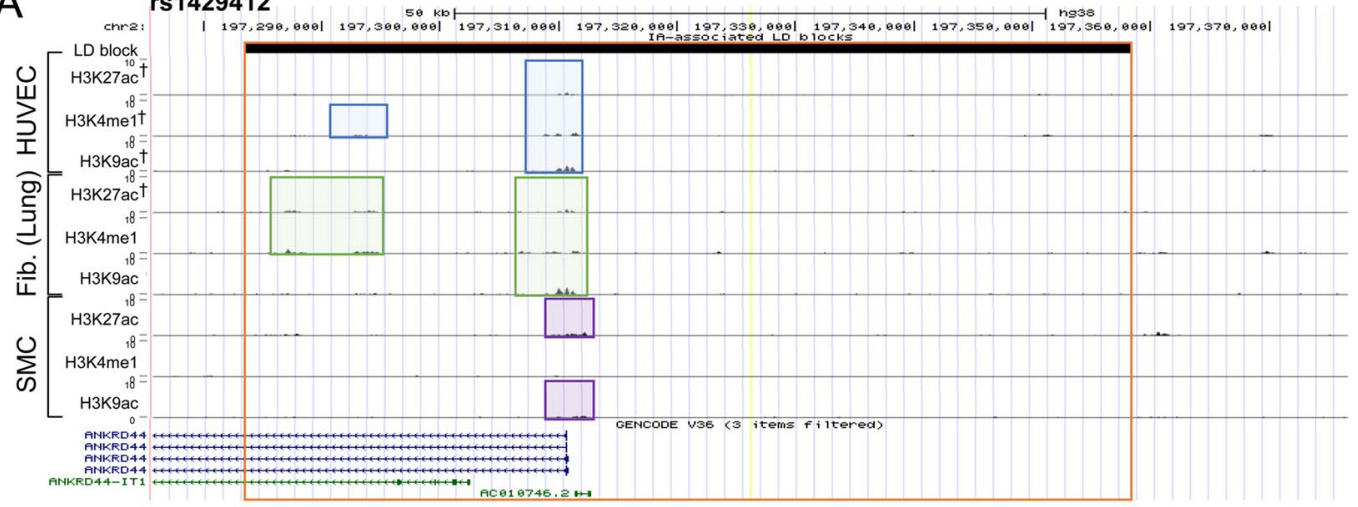

B

rs1800796

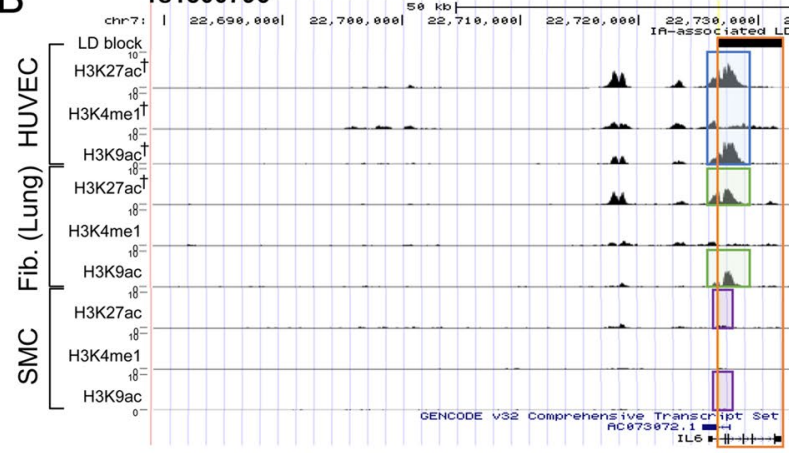

C

rs10757278

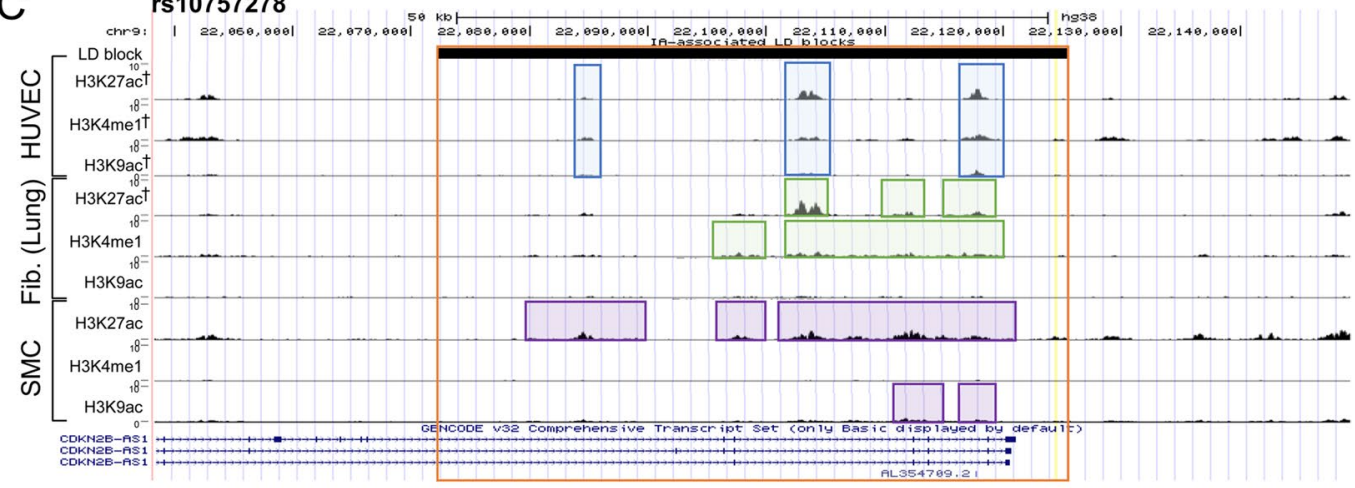

D

rs6538595

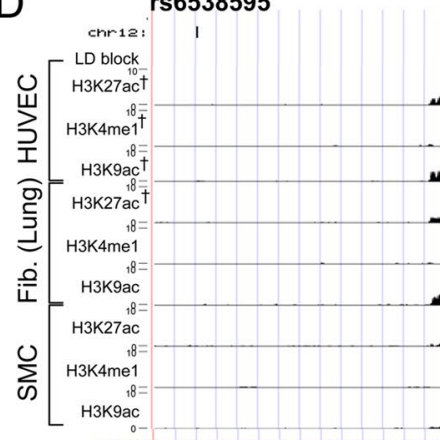

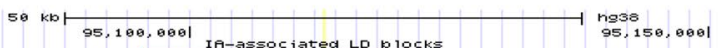

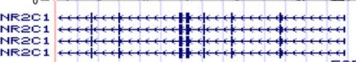

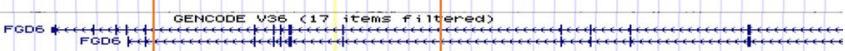


(See figure on next page.)

Fig. 2 Visualization of the topologically associated domain (TAD) of four example SNPs in HUVECs. Haplotype outlined in red. TAD outlined in black. Genes reported as differentially expressed in IA tissue studies are highlighted in red (increased expression) or green (decreased expression). Border indicates identified in IA vs control; fill indicates identified in ruptured vs unruptured IAs. A HiC data surrounding rs 1429412 shows a large TAD in HUVECs. B Data from HiC analysis shows another large TAD surrounding rs 1800796. C HiC data shows the TAD surrounding rs 10757278. D HiC map showing TAD encompassing rs6538595 does not contain any genes found to be differentially expressed in IA tissue studies

TADs were reported as differentially expressed in IA tissue studies.

\section{Discussion}

In this study, we mined publicly-available data from the ENCODE database to gain a better understanding of the nature of genetic risk for IA and how it is related to transcriptional abnormalities observed in human aneurysmal tissue. To do this, we investigated epigenetic landscapes of 16 validated IA risk haplotypes in cells found in the walls of IAs. From ChIP-Seq data, we found evidence that the risk haplotypes defined by the GWAS tag SNPs were enriched for H3K4me1, H3K27ac, and H3K9ac marks, (marks typically associated with enhancer activity [52]), and that these putative regulatory elements were present to a significantly greater degree in the IA-associated risk regions in ECs and fibroblasts rather than other cell types that reside in human IA tissues. While these results are similar to that of our previous work [18], we were able to perform several complementary analyses in this study to gain further insight about epigenetic regulation of gene expression in IA. Indeed, we studied the broader chromatin architecture encompassing the haplotypes that demonstrated H3K4me1/H3K27ac/H3K9ac enrichment, specifically focusing on $\mathrm{HiC}$-defined chromatin loops (i.e. TADs), which are known to facilitate specific enhancerpromoter interactions while limiting others [53]. Bioinformatics analysis of these TADs in HUVECs and IMR90 fibroblasts identified genes plausibly related to IA pathogenesis. A total of 25 of the genes within these TADs have been independently shown to be differentially expressed in human IA tissues. These findings corroborate the idea that genetic modification of enhancers on the risk haplotypes may directly alter gene expression, although the specific enhancer-promoter interactions mediating these effects cannot be ascertained from these types of data. Furthermore, our use of multiple data sources helps to reduce the number of promising target genes through which disease-associated variants may act. We began with those identified in IA-risk associated TADs (164 in HUVECs, 153 in IMR90 fibroblasts) and refined to those genes that also have reported differential expression in IA tissue (20 in HUVECs, 19 in IMR90 cells).

In our recent study [18], we explored the chromatin landscapes associated with these same 16 haplotypes that were reported in a meta-analysis by Alg et al. [19], in order to assess whether there was epigenetic evidence that genetic risk was conferred more predominantly in circulating immune cells or the vascular endothelium. There, we demonstrated that significant enrichment for H3K4me1 and H3K27ac histone peaks was observed exclusively in endothelial cells, compared to peripheral blood leukocytes [18]. Perturbations in endothelial gene expression are thought to be among the first pathobiological vascular responses that precede IA formation [54-57]. Thus, these results had indicated that genetic risk for IA may be conferred through the vascular wall rather than circulating immune cells, which prompted the current study's investigation of additional cell types found in arterial tissues, as well as broader chromatin architecture that subsumes the risk haplotypes. The current study corroborates our earlier finding that HUVECs showed significant enrichment for H3K4me1 (present in 13/16 LD blocks, $p=0.00025$ ) and H3K27ac (present in 9/16 LD blocks, $p=0.0031$ ) ChIP-Seq peaks, since the same list of 16 LD blocks and same ChIP-Seq dataset was used. In that study, the sites marked by H3K4me1 and H3K27ac in HUVECs had also demonstrated abundant transcription factor binding sites and CTCF binding sites, further signifying that these regions were associated with functional enhancer activity.

In the current study, we were able to perform additional analyses using H3K9ac ChIPseq data, another indicator of functionally active chromatin [52, 58, 59]. We found that this mark demonstrated significant enrichment $(p=0.014)$ and was present in 7 of the LD blocks for HUVECs. We also investigated ChIP-Seq data from HAECs, an endothelial cell line that may be more biologically relevant to the arterial vasculature where IAs form than venous cells from the umbilical cord. However, we were only able to show significant enrichment of H3K27ac marks (present in 10/16 LD blocks, $p=0.01$ ), as ChIP-Seq data was unavailable for H3K4me1 and H3K9ac. Taken together, these data yet again implicate ECs, which have been widely recognized as a key player in IA natural history $[55,56,60]$, as a main cell type to be most likely affected by genetic risk for IA.

In addition to ECs, the other cell type we investigated that had significant enrichment for the ChIP-Seq peaks of interest were fibroblasts. While not extensively studied in IA, fibroblasts are known to coordinate the production of collagen (particularly Type I), as well as produce 
A

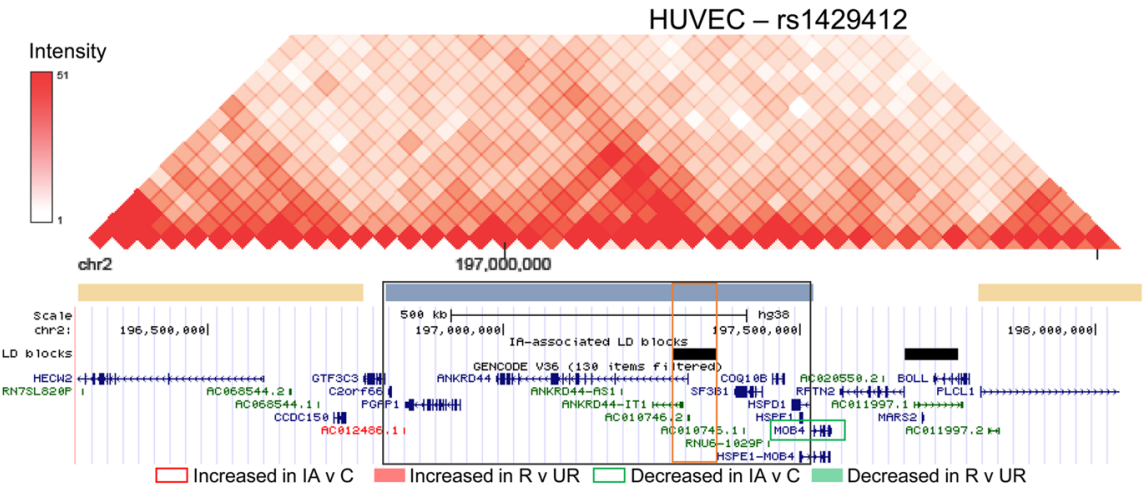

B
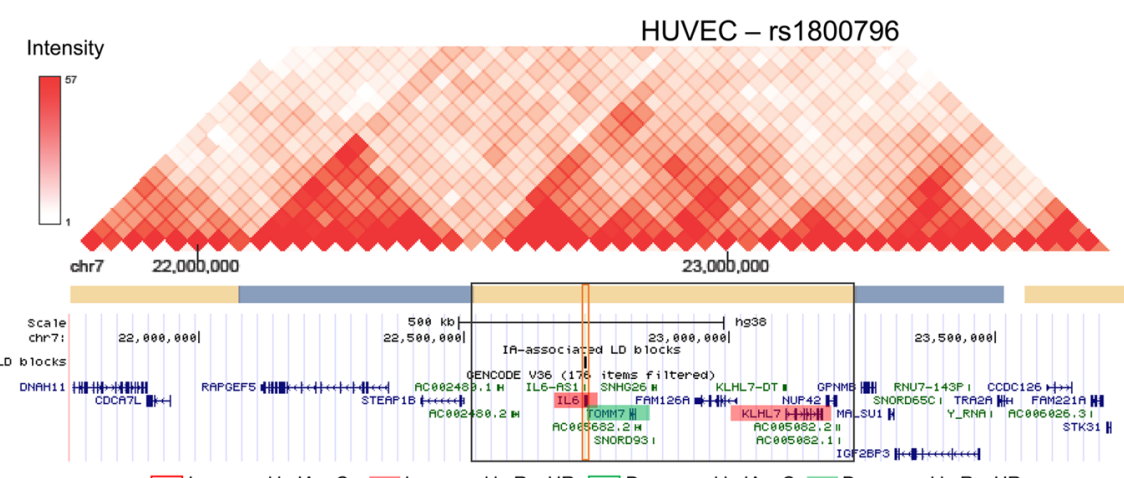

C

$\square$ Increased in IA v C $\quad$ Increased in R v UR $\square$ Decreased in IA v C Decreased in R v UR

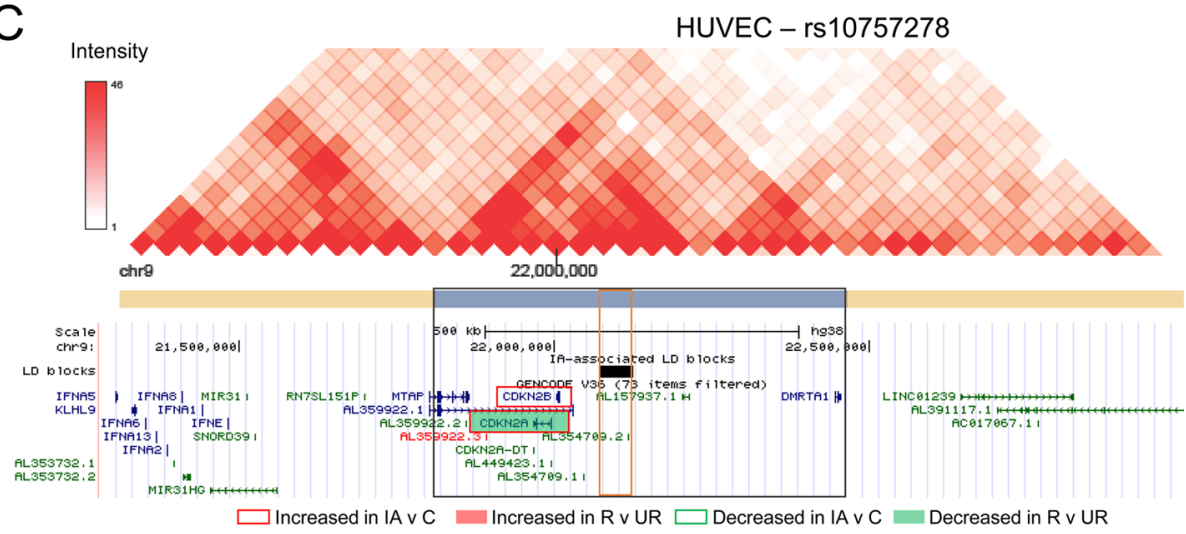

D

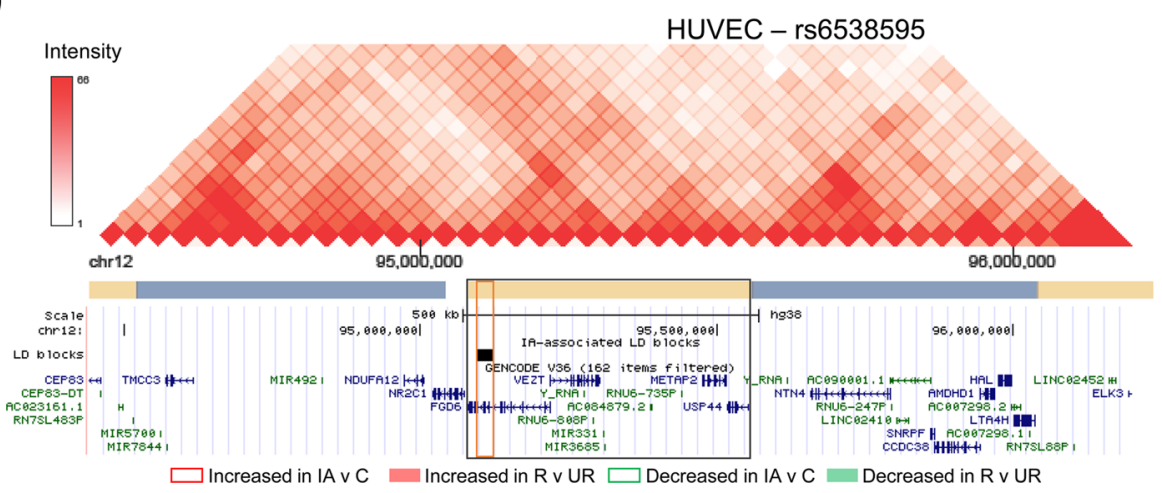




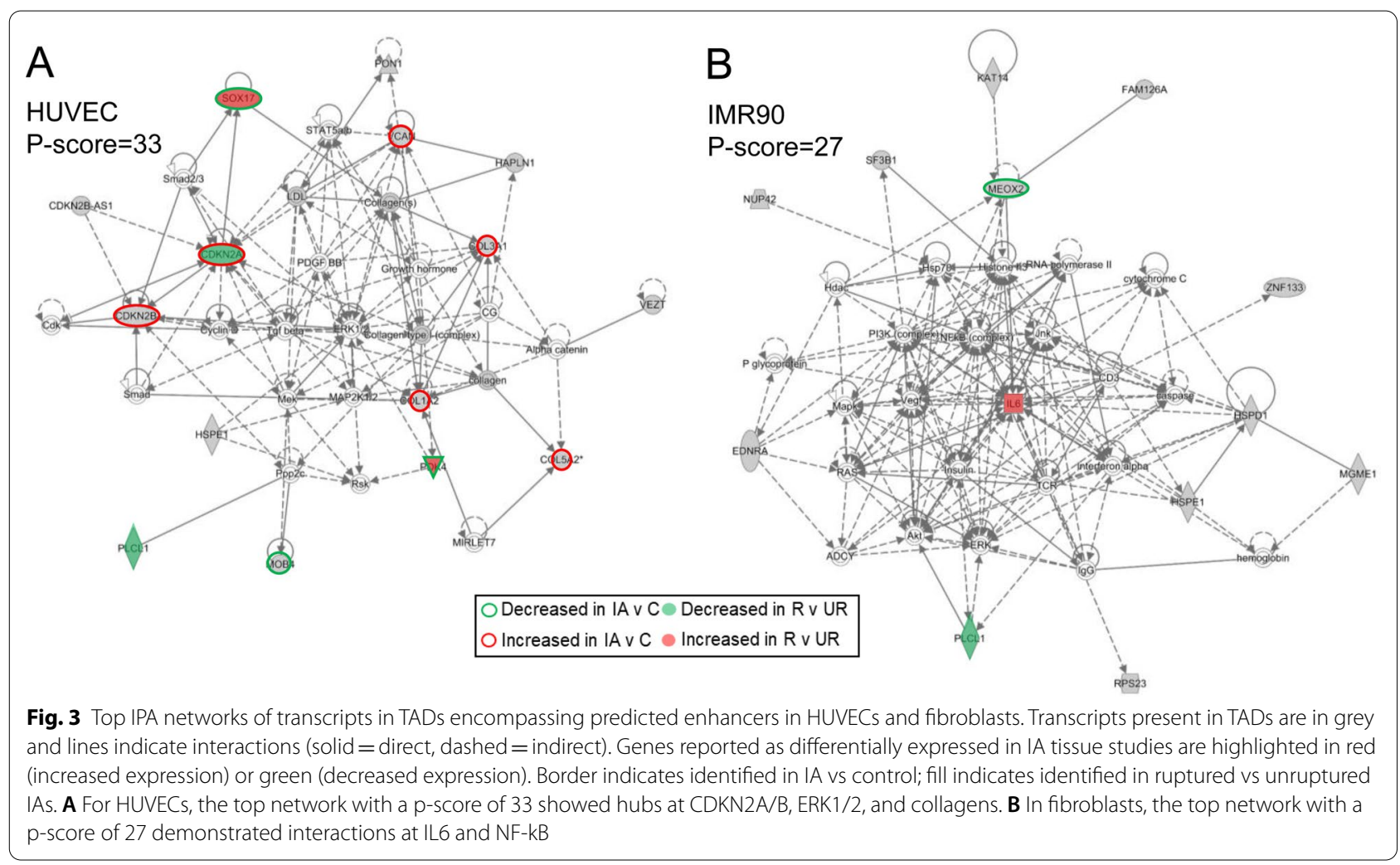

and organize extracellular matrix, where they resize and carry out adherent and contractile abilities [61]. Additionally, they can produce matrix metalloproteinases, which facilitate vessel wall destruction and remodeling during IA formation $[62,63]$. In our data, skin fibroblasts showed significant enrichment in H3K4me1 (present in 7/16 LD blocks, $p=0.0091$ ), H3K27ac (present in 9/16 LD blocks, $p=0.0027$ ), and H3K9ac marks (present in $6 / 16$ LD blocks, $p=0.010$ ), while lung fibroblasts only showed enrichment of H3K27ac (present in 8/16 LD blocks, $p=0.0079$ ). Therefore, it appears that, in addition to ECs, fibroblasts are another cellular component of the vascular wall where the biological effects of genetic variants may confer risk for the development of IA.

To identify genes potentially affected by enhancers in the LD blocks investigated in this study, we examined the TADs (regions of the genome that form interactions and thereby affect gene expression together) encompassing the 16 LD blocks of interest. For ECs, ontology annotations associated with genes within the TADs reflected extracellular matrix structure and enzymatic activity. Furthermore, disease and function annotation in IPA showed significant enrichment of Loeys-Dietz syndrome, Ehlers-Danlos syndrome, and vascular lesion. The bioinformatics results broadly reflect the connective tissue weakening that is central to the pathogenesis and progression of IA. This is further reflected in hub genes of significant networks, including multiple forms of collagen and VCAN, or versican, a proteoglycan that is a major component of the extracellular matrix of vessels.

In associated TADs in fibroblasts, there was a large degree of overlap between genes found within HUVECs. Therefore, it is not surprising that bioinformatics performed on this group of genes also reflected phosphorylase activity. Additionally, IPA networks of gene interactions reflected many hub molecules identified in our analysis of ECs, i.e. collagens and VCAN. However, they also reflected immune responses, with hubs at Akt, ERK1/2, and NF-kB (all transcription factors) in addition to a large node at IL6. NF-kB is an important mediator of gene expression in IA formation that can regulate the expression of may interleukins, cytokines, and chemokines known to play key roles in IA natural history.

It is important to recognize that enhancer function cannot be determined on the basis of chromatin marks alone. Therefore, to further assess if genes located in TADs subsuming the IA-associated LD blocks in ECs and fibroblasts were, indeed, differentially expressed in IA tissue, we compared our list of potential targets to differentially expressed genes reported in numerous studies (see Table 3). In studies that compared control 
Table 3 Differentially expressed genes in IA tissue present within IA risk associated TADs

\begin{tabular}{|c|c|c|c|c|}
\hline \multirow[b]{2}{*}{ Gene (TAD found in) } & \multicolumn{2}{|l|}{ HUVEC TAD } & \multicolumn{2}{|c|}{ Fib. (IMR90) TAD } \\
\hline & $I A$ versus $C t r$ & $R$ versus $U R$ & $I A$ versus $C t r$ & $R$ versus $U R$ \\
\hline \multirow[t]{2}{*}{ BFSP1 (rs1132274) } & & & & Kleinloog $(\downarrow,-4.0)$ \\
\hline & & & & Nakaoka $(\downarrow,-1.8)$ \\
\hline CASD1 (rs42524) & & Kurki $(\downarrow,-1.0)$ & & \\
\hline CDKN2A (rs2891168, rs 10757278) & Wang (, NR) & $\operatorname{Nakaoka~}(\downarrow,-1.9)$ & Wang $(\uparrow, N R)$ & $\operatorname{Nakaoka}(\downarrow,-1.9)$ \\
\hline CDKN2B (rs2891 168, rs10757278) & Wang $(\uparrow, N R)$ & & Wang $(\uparrow, N R)$ & \\
\hline \multirow[t]{2}{*}{ COL1A2 (rs42524) } & $\mathrm{Li}(\uparrow, N R)$ & & $\mathrm{Li}(\uparrow, N R)$ & \\
\hline & Wang $(\uparrow, N R)$ & & Wang $(\uparrow, N R)$ & \\
\hline \multirow[t]{2}{*}{ COL3A1 (rs1800255) } & $\mathrm{Li}(\uparrow, N R)$ & & $\mathrm{Li}(\uparrow, N R)$ & \\
\hline & Wang $(\uparrow, N R)$ & & Wang $(\uparrow, N R)$ & \\
\hline \multirow[t]{3}{*}{ COL5A2 (rs1800255) } & $\mathrm{Li}(\uparrow, N R)$ & & & \\
\hline & Shi $(\uparrow, 3.3)$ & & & \\
\hline & Wang $(\uparrow, N R)$ & & & \\
\hline DSTN (rs1132274) & & & Wang $(\downarrow, N R)$ & \\
\hline \multirow[t]{2}{*}{ GULP1 (rs1800255) } & & Kurki $(\downarrow,-2.2)$ & & Kurki $(\downarrow,-2.2)$ \\
\hline & & $\operatorname{Nakaoka}(\downarrow,-1.3)$ & & $\operatorname{Nakaoka}(\downarrow,-1.3)$ \\
\hline IL6 (rs1800796) & & $\operatorname{Kurki}(\uparrow, 2.2)$ & & Kurki $(\uparrow, 2.2)$ \\
\hline KLHL7 (rs 1800796) & & Nakaoka $(\uparrow, 0.50)$ & & Nakaoka $(\uparrow, 0.50)$ \\
\hline MEOX2 (rs4628172) & Wang $(\downarrow, N R)$ & & Wang $(\downarrow, N R)$ & \\
\hline MOB4 (rs1429412) & Wang $(\downarrow, N R)$ & & Wang $(\downarrow, N R)$ & \\
\hline PDK4 (rs42524) & Wang $(\downarrow, N R)$ & $\operatorname{Kurki}(\uparrow, 1.0)$ & & \\
\hline \multirow[t]{2}{*}{ PLCL1 (rs700651) } & & Kleinloog $(\downarrow,-4.0)$ & & Kleinloog $(\downarrow,-4.0)$ \\
\hline & & Kurki $(\downarrow,-1.4)$ & & Kurki $(\downarrow,-1.4)$ \\
\hline PPP1R9A (rs42524) & Wang $(\downarrow, N R)$ & & & \\
\hline RRBP1 (rs1132274) & & & Wang $(\uparrow, N R)$ & \\
\hline SGCE (rs42524) & Wang $(\downarrow, N R)$ & Kurki $(\downarrow,-2.3)$ & & \\
\hline SNX5 (rs1132274) & & & Wang $(\uparrow, N R)$ & \\
\hline SOX17 (rs10958409, rs9598506) & Wang $(\downarrow, N R)$ & Nakaoka $(\uparrow, 0.88)$ & & \\
\hline TFPI (rs1800255) & & & Wang $(\downarrow, N R)$ & \\
\hline TMEM167A (rs251124) & & Kurki $(\uparrow, 1.2)$ & & Kurki $(\uparrow, 1.2)$ \\
\hline TOMM7 (rs1800796) & & Kurki $(\downarrow,-0.86)$ & & $\operatorname{Kurki}(\downarrow,-0.86)$ \\
\hline \multirow[t]{2}{*}{ VCAN (rs251124) } & Shi $(\uparrow, 2.9)$ & & Shi $(\uparrow, 2.9)$ & \\
\hline & Wang $(\uparrow, N R)$ & & Wang $(\uparrow, N R)$ & \\
\hline XRCC4 (rs251124) & & Kleinloog $(\uparrow, 2.3)$ & & Kleinloog $(\uparrow, 2.3)$ \\
\hline
\end{tabular}

The $\log _{2}$ (fold-change) for each gene as reported in the respective study is shown in parentheses. If study did not provide fold-change, NR is reported. Multiple probes corresponded to Nakaoka's CDKN2A; average value was reported

Ctr., control; Fib., fibroblast; HUVEC, human umbilical vein endothelial cell; IA, intracranial aneurysm; NR, not reported; R, ruptured; TAD, topologically associated domain; UR, unruptured

vascular tissue (primarily superior temporal artery tissue), HUVEC and fibroblast TAD genes included structural collagens, COL1A2 and COL3A1, which were found to be increased in IA tissue. The inclusion of $V C A N$, which also was increased in IA tissue, further reflects the role of dysregulated extracellular matrix components, as it plays a role in cell adhesion, proliferation, migration, and angiogenesis [64]. Differential expression of $C D K N 2 A / B$, a gene that is involved in regulating $\mathrm{EC}$ proliferation and repair in an effort to prevent vascular injury [12], was also present in the HUVEC and fibroblast TAD genes, as was MEOX2, a transcription factor that activates the expression of $C D K N 2 A$ [65]. Multiple GWAS show that an exonic SNP in CDKN2 (9p213) is highly associated with IA. However, our results may indicate that this gene could also be regulated by aberrant enhancer activity. Ultimately, these data support the idea that genetic risk is exerted predominantly on extracellular matrix processes carried out by vascular ECs and fibroblasts. 
Studies that compared ruptured IA tissue to unruptured IA tissue shared many of these same genes that were also present in HUVEC and fibroblast TADs. There were several genes that were uniquely differentially expressed when unruptured IAs were compared to ruptured ones. KLHL7, which may participate in protein degradation, is overexpressed in ruptured IA cases. IL6, also increased in ruptured cases, is a proinflammatory cytokine that has long been recognized in IA pathology [66]. IL6 promoter polymorphisms, such as rs1800796, are also associated with IA and other inflammatory diseases [67]. Consequently, we suspect that in addition to extracellular matrix processes, genetic risk may also affect inflammatory responses in the vessel wall. Interestingly, the effect of SNPs on expression of IL6 may be similar to that on CDKN2. Both regions containing prominent histone marks within the gene, which may function as so-called "exonic enhancers" [68]. In such a case, the genetic variant could operate through the gene sequence function, the enhancer function, or both to lead to differential expression of the gene. Taken together, our results show strong support that the studied regions contain active enhancers, in which disruption by SNPs causes aberrant changes in gene expression. However, experimental studies empirically demonstrating the effect of altered enhancer activity of IA-associated variants is still needed.

Our study has several limitations. First, we focused on SNPs identified in one meta-analysis. While this meta-analysis was comprehensive and the SNPs identified were found in at least two studies that analyzed a large volume of cases in controlled populations, a larger study in the future including SNPs from other works would be beneficial. Second, we generalized our results from HUVECs and SMCs to IA samples, though these cell types may be different in arterial aneurysm tissue. When possible, we used cell types that more reflected those in IA walls, such as HAECs instead of HUVECs. However, in many cases we were limited by data availability, i.e. there was not as much $\mathrm{Hi}-\mathrm{C}$ data available for our TAD analysis as we would have liked. For example, HUVECs had widely-available datasets, but fibroblasts did not, and thus we resorted to using data from IMR90 cells instead of skin fibroblasts for TAD analysis. We are currently planning experimental validations of these findings in cell lines more similar to IA-resident cells. Lastly, we recognize that gene expression studies in IA tissue report differential expression that is aggregated from the many cell types that are present in the aneurysm. Single-cell studies would be needed to verify differential expression of genes within IA-associated TADs from one cell type vs. another.

\section{Conclusions}

We analyzed H3K4me1, H3K27ac, and H3K9ac histone marks in 16 IA-risk regions, reporting that genetic risk for IA is conferred through ECs and fibroblasts. Our findings provide evidence that genetic variants known to be associated with IA risk act on endothelial cells and fibroblasts, rather than immune cells or smooth muscle cells. Based on analysis of genes in overlapping TADs, genetic risk of IA may affect regulation of genes involved in extracellular matrix integrity in ECs and fibroblasts. There is strong circumstantial evidence that this may be mediated through altered enhancer function, as multiple genes in TADs encompassing the enhancer marks have also been shown to be differentially expressed in the IA tissue.

\section{Abbreviations}

DEG: Differentially expressed gene; EC: Endothelial cell; GO: Gene ontology; GWAS: Genome-wide association study; HAEC: Human aortic endothelial cell; HUVEC: Human umbilical vein endothelial cell; IA: Intracranial aneurysm; IPA: Ingenuity pathway analysis; LD: Linkage disequilibrium; NK: Natural killer; OR: Odds ratio; PBMC: Peripheral blood mononuclear cell; PNL: Polymorphonuclear leukocyte; SMC: Smooth muscle cell; SNP: Single nucleotide polymorphism; TAD: Topologically associated domain.

\section{Supplementary Information}

The online version contains supplementary material available at https://doi. org/10.1186/s12920-021-01007-9.

Additional file 1: Fig. S1. Visualization of the topologically associated domains (TADs) of four example SNPs in fibroblasts (IMR90 cells). Tiff image. Fig. S2. Additional IPA networks of transcripts in TADs encompassing predicted enhancers in HUVECs and fibroblasts. Tiff image. Table S1. Cistrome datasets for histone mark analysis. Table S2. Conversion of IA associated linkage disequilibrium blocks to hg38. Table S3. Genes encompassed within TADs surrounding IA-risk associated haplotypes. Table S4. gProfiler GO ontologies of genes within IA associated, histone marked TADs. Table S5. IPA diseases and biological functions of genes within IA associated, histone marked TADs. Table S6. IPA Networks of genes within IA associated, histone marked TADs. Table S7. IA tissue differential expression studies.

\section{Acknowledgements}

None.

\section{Authors' contributions}

KEP, JNJ, VMT conceived and designed the study. KEP, HRZ, NA, VMT analyzed the data and performed the statistical analyses. KEP, JNJ, VMT drafted the manuscript. All authors read and approved the final manuscript.

\section{Funding}

This research was supported by the Brain Aneurysm Foundation (VMT) and the State of New York through the University at Buffalo's Center for Advanced Technologies in Big Data and Health Sciences (VMT). The opinions, results, findings and/or interpretations are the sole responsibility of the authors and do not represent the opinions, interpretations or policy of the funders.

\section{Availability of data and materials}

The GEO/ENCODE IDs for the datasets obtained through Cistrome database [http://cistrome.org/db/\#/] are as follows: NK cell H3K27ac - GSM999008, H3K4me1 - GSM999007; CD4 + T cell H3K27ac - GSM1220560, H3K4me1 GSM1220567, H3K9ac - GSM543004; CD8 + T cell H3K27ac - GSM1 102781, 
H3K4me1 - GSM1220569, H3K9ac - GSM613813; SMC H3K27ac - ENCSR210ZPC_2, H3K4me1 - ENCSR130IMV_2, H3K9ac - ENCSR540UZV_2; GM12878 cell H3K27ac - GSM733771, H3K4me1 - GSM733772, H3K9ac - GSM733677; HUVEC H3K27ac - GSM733691, H3K4me1 - GSM733690, H3K9ac GSM733735, H3K9me3 - GSM1003517; ung fibroblast H3K27ac - GSM733646, H3K4me1 - GSM733649, H3K9ac - GSM733652, H3K9me3 - GSM733695; Skin fibroblast H3K27ac - GSM733662, H3K4me1 - GSM1003526, H3K9ac GSM733709, H3K9me3 - GSM733744; Monocyte H3K27ac - GSM1003559, H3K4me1 - GSM1003535, H3K9ac - GSM1003515; Neutrophil H3K27ac - GSM2527660, H3K4me1 - GSM2534489; HAEC H3K27ac - GSM2394402; Macrophage H3K27ac - GSM785500, H3K4me1 - GSM785498; The GEO ID for the dataset used in the 3D genome browser [http://3dgenome.fsm.north western.edu/view.php] is GSE63525.

\section{Declarations}

\section{Ethics approval and consent to participate}

Not applicable.

\section{Consent for publication}

Not applicable.

\section{Competing interests}

KEP, HRZ, NA, MW—None. AHS—Financial Interest/Investor/Stock Options/ Ownership: Amnis Therapeutics, Apama Medi-cal, BlinkTBI, Inc, Buffalo Technology Partners, Inc., Cardinal Health, Cerebrotech Medical Systems, Inc, Claret Medical, Cognition Medical, Endostream Medical, Ltd, Imperative Care, International Medical Distribution Partners, Rebound Therapeutics Corp., Silk Road Medical, StimMed, Synchron, Three Rivers Medi- cal, Inc., Viseon Spine, Inc. Consultant/Advisory Board: Amnis Therapeutics, Boston Scientific, Canon Medical Systems USA, Inc., Cerebrotech Medical Systems, Inc., Cerenovus, Claret Medical, Corindus, Inc., Endostream Medical, Ltd, Guidepoint Global Consulting, Imperative Care, Integra, Medtronic, Micro- Vention, Northwest University—DSMB Chair for HEAT Trial, Penumbra, Rapid Medical, Rebound Therapeutics Corp., Silk Road Medical, StimMed, Stryker, Three Rivers Medical, Inc., VasSol, W.L. Gore \& Associates. National PI/Steering Committees: Cerenovus LARGE Trial and ARISE II Trial, Medtronic SWIFT PRIME and SWIFT DIRECT Trials, MicroVention FRED Trial \& CONFIDENCE Study, MUSC POSITIVE Trial, Penumbra 3D Separator Trial, COMPASS Trial, INVEST Trial. Principal investigator: Cummings Foundation grant. JNJ_Principal Investigator: R21-AR071878. VMT_Principal investigator: National Science Foundation Award No. 1746694 and NIH NINDS award R43 NS115314-0. Grant funding from: Brain Aneurysm Foundation grant, Centers for Advanced Technology grant (through NY Empire State Development), and Cummings Foundation. Co-founder: Neurovascular Diagnostics, Inc.

\section{Author details}

${ }^{1}$ Canon Stroke and Vascular Research Center, University at Buffalo, Clinical and Translational Research Center, 875 Ellicott Street, Buffalo, NY 14214, USA. ${ }^{2}$ Department of Neurosurgery, University at Buffalo, Buffalo, NY, USA. ${ }^{3}$ Department of Biomedical Engineering, University at Buffalo, Buffalo, NY, USA. ${ }^{4}$ Department of Pediatrics, University at Buffalo, Buffalo, NY, USA. ${ }^{5}$ Department of Pathology and Anatomical Sciences, University at Buffalo, Buffalo, NY, USA. ${ }^{6}$ Department of Mechanical and Aerospace Engineering, University at Buffalo, Buffalo, NY, USA.

Received: 10 December 2020 Accepted: 2 June 2021 Published online: 16 June 2021

\section{References}

1. Vega C, Kwoon JV, Lavine SD. Intracranial aneurysms: current evidence and clinical practice. Am Fam Physician. 2002;66(4):601-8.

2. Ronkainen A, Hernesniemi J, Puranen M, Niemitukia L, Vanninen R, Ryynänen M, et al. Familial intracranial aneurysms. Lancet. 1997;349(9049):380-4.

3. Okamoto K, Horisawa R, Kawamura T, Asai A, Ogino M, Takagi T, et al. Family History and Risk of Subarachnoid Hemorrhage. Stroke. 2003:34(2):422-6.
4. Abrantes P, Santos MM, Sousa I, Xavier JM, Francisco V, Krug T, et al. Genetic variants underlying risk of intracranial aneurysms: insights from a GWAS in Portugal. PLoS ONE. 2015;10(7):e0133422.

5. Deka R, Koller DL, Lai D, Indugula SR, Sun G, Woo D, et al. The relationship between smoking and replicated sequence variants on chromosomes 8 and 9 with familial intracranial aneurysm. Stroke. 2010;41(6):1132-7.

6. Foroud T, Koller DL, Lai D, Sauerbeck L, Anderson C, Ko N, et al. Genomewide association study of intracranial aneurysms confirms role of Anril and SOX17 in disease risk. Stroke. 2012;43(11):2846-52.

7. Foroud T, Lai D, Koller D, Van't Hof F, Kurki Ml, Anderson CS, et al. Genomewide association study of intracranial aneurysm identifies a new association on chromosome 7. Stroke. 2014;45(11):3194-9.

8. Kurki MI, Gaal El, Kettunen J, Lappalainen T, Menelaou A, Anttila V, et al. High risk population isolate reveals low frequency variants predisposing to intracranial aneurysms. PLoS Genet. 2014;10(1):e1004134.

9. Low SK, Takahashi A, Cha PC, Zembutsu H, Kamatani N, Kubo M, et al. Genome-wide association study for intracranial aneurysm in the Japanese population identifies three candidate susceptible loci and a functional genetic variant at EDNRA. Hum Mol Genet. 2012;21(9):2102-10.

10. Yasuno K, Bakircioglu M, Low SK, Bilguvar K, Gaal E, Ruigrok YM, et al. Common variant near the endothelin receptor type A (EDNRA) gene is associated with intracranial aneurysm risk. Proc Natl Acad Sci USA. 2011;108(49):19707-12.

11. Yasuno K, Bilguvar K, Bijlenga P, Low SK, Krischek B, Auburger G, et al. Genome-wide association study of intracranial aneurysm identifies three new risk loci. Nat Genet. 2010;42(5):420-5.

12. Bilguvar K, Yasuno K, Niemelä M, Ruigrok YM, von Und Zu, Fraunberg M, van Duijn CM, et al. Susceptibility loci for intracranial aneurysm in European and Japanese populations. Nat Genet. 2008;40(12):1472-7.

13. Bulger M, Groudine M. Enhancers: the abundance and function of regulatory sequences beyond promoters. Dev Biol. 2010;339(2):250-7.

14. Martin P, McGovern A, Orozco G, Duffus K, Yarwood A, Schoenfelder S, et al. Capture Hi-C reveals novel candidate genes and complex longrange interactions with related autoimmune risk loci. Nat Commun. 2015:6:10069. https://doi.org/10.1038/ncomms10069.

15. Stram DO. Tag SNP selection for association studies. Genet Epidemiol. 2004;27(4):365-74.

16. Ding K, Kullo IJ. Methods for the selection of tagging SNPS: a comparison of tagging efficiency and performance. Eur J Hum Genet EJHG. 2007;15(2):228-36.

17. MacArthur DG, Manolio TA, Dimmock DP, Rehm HL, Shendure J, Abecasis $\mathrm{GR}$, et al. Guidelines for investigating causality of sequence variants in human disease. Nature. 2014;508(7497):469-76.

18. Poppenberg KE, Jiang K, Tso MK, Snyder KV, Siddiqui AH, Kolega J, et al. Epigenetic landscapes suggest that genetic risk for intracranial aneurysm operates on the endothelium. BMC Med Genom. 2019;12(1):149.

19. Alg VS, Sofat R, Houlden H, Werring DJ. Genetic risk factors for intracranial aneurysms: a meta-analysis in more than 116,000 individuals. Neurology. 2013;80(23):2154-65.

20. Zentner GE, Tesar PJ, Scacheri PC. Epigenetic signatures distinguish multiple classes of enhancers with distinct cellular functions. Genome Res. 2011;21(8):1273-83.

21. Gasperini M, Hill AJ, McFaline-Figueroa JL, Martin B, Kim S, Zhang MD, et al. A genome-wide framework for mapping gene regulation via cellular genetic screens. Cell. 2019;176(1):377-90.e19.

22. Frösen J, Piippo A, Paetau A, Kangasniemi M, Niemelä M, Hernesniemi J, et al. Remodeling of saccular cerebral artery aneurysm wall is associated with rupture. Stroke. 2004;35(10):2287.

23. Chyatte D, Bruno G, Desai S, Todor DR. Inflammation and intracranial aneurysms. Neurosurgery. 1999;45(5):1137-47.

24. KosierkiewiczTA, Factor SM, Dickson DW. Immunocytochemical studies of atherosclerotic lesions of cerebral berry aneurysms. J Neuropathol Exp Neurol. 1994;53(4):399-406.

25. Marbacher S, Marjamaa J, Bradacova K, von Gunten M, Honkanen P, Abo-Ramadan U, et al. Loss of mural cells leads to wall degeneration, aneurysm growth, and eventual rupture in a rat aneurysm model. Stroke. 2014;45(1):248-54.

26. Kataoka K, Taneda M, Asai T, Kinoshita A, Ito M, Kuroda R. Structural fragility and inflammatory response of ruptured cerebral aneurysms. A comparative study between ruptured and unruptured cerebral aneurysms. Stroke. 1999;30(7):1396-401. 
27. Rao SSP, Huntley MH, Durand NC, Stamenova EK, Bochkov ID, Robinson JT, et al. A 3D map of the human genome at kilobase resolution reveals principles of chromatin looping. Cell. 2014;159(7):1665-80.

28. Mei S, Qin Q, Wu Q, Sun H, Zheng R, Zang C, et al. Cistrome Data Browser: a data portal for ChIP-Seq and chromatin accessibility data in human and mouse. Nucleic Acids Res. 2017:45(D1):D658-62.

29. Zheng R, Wan C, Mei S, Qin Q, Wu Q, Sun H, et al. Cistrome Data Browser: expanded datasets and new tools for gene regulatory analysis. Nucleic Acids Res. 2019;47(D1):D729-35.

30. Qin Q, Mei S, Wu Q, Sun H, Li L, Taing L, et al. ChiLin: a comprehensive ChIP-seq and DNase-seq quality control and analysis pipeline. BMC Bioinformatics. 2016;17(1):404.

31. Kataoka K, Taneda M, Asai T, Kinoshita A, Ito M, Kuroda R. Structural fragility and inflammatory response of ruptured cerebral aneurysms. Stroke. 1999;30(7):1396.

32. Frösen J, Tulamo R, Paetau A, Laaksamo E, Korja M, Laakso A, et al. Saccular intracranial aneurysm: pathology and mechanisms. Acta Neuropathol. 2012;123(6):773-86.

33. Frösen J, Joutel A. Smooth muscle cells of intracranial vessels: from development to disease. Cardiovasc Res. 2018;114(4):501-12.

34. Wang Y, Song F, Zhang B, Zhang L, Xu J, Kuang D, et al. The 3D Genome Browser: a web-based browser for visualizing 3D genome organization and long-range chromatin interactions. Genome Biol. 2018;19(1):151.

35. Belton J-M, McCord RP, Gibcus JH, Naumova N, Zhan Y, Dekker J. Hi-C: a comprehensive technique to capture the conformation of genomes. Methods (San Diego, Calif). 2012;58(3):268-76.

36. Raudvere U, Kolberg L, Kuzmin I, ArakT, Adler P, Peterson H, et al. g:Profiler: a web server for functional enrichment analysis and conversions of gene lists (2019 update). Nucleic Acids Res. 2019;47(W1):W191-8.

37. Krämer A, Green J, Pollard J Jr, Tugendreich S. Causal analysis approaches in ingenuity pathway analysis. Bioinformatics (Oxford, England). 2014;30(4):523-30.

38. Aoki T, Koseki H, Miyata H, Itoh M, Kawaji H, Takizawa K, et al. RNA sequencing analysis revealed the induction of CCL3 expression in human intracranial aneurysms. Sci Rep. 2019;9(1):10387.

39. Kleinloog R, Verweij Bon H, van der Vlies P, Deelen P, Swertz Morris A, de Muynck $L$, et al. RNA sequencing analysis of intracranial aneurysm walls reveals involvement of lysosomes and immunoglobulins in rupture. Stroke. 2016;47(5):1286-93.

40. Li L, Yang X, Jiang F, Dusting GJ, Wu Z. Transcriptome-wide characterization of gene expression associated with unruptured intracranial aneurysms. Eur Neurol. 2009;62(6):330-7.

41. Pera J, Korostynski M, Krzyszkowski T, Czopek J, Slowik A, Dziedzic T, et al. Gene expression profiles in human ruptured and unruptured intracranial aneurysms. Stroke. 2010;41(2):224.

42. Shi C, Awad IA, Jafari N, Lin S, Du P, Hage ZA, et al. Genomics of human intracranial aneurysm wall. Stroke. 2009;40(4):1252.

43. Wang W, Li H, Yu L, et al. Aberrant expression of IncRNAs and mRNAs in patients with intracranial aneurysm. Oncotarget. 2017;8(2):2477-484. https://doi.org/10.18632/oncotarget.13908.

44. Kurki MI, Häkkinen S-K, Frösen J, Tulamo R, von und zu Fraunberg M Wong $\mathrm{G}$, et al. Upregulated signaling pathways in ruptured human saccular intracranial aneurysm wall: an emerging regulative role of toll-like receptor signaling and nuclear factor-kB, hypoxia-inducible factor-1A, and ETS transcription factors. Neurosurgery. 2011;68(6):1667-76.

45. Nakaoka H, Tajima A, Yoneyama T, Hosomichi K, Kasuya H, Mizutani T, et al. Gene expression profiling reveals distinct molecular signatures associated with the rupture of intracranial aneurysm. Stroke. 2014;45(8):2239.

46. Ahituv N. Exonic enhancers: proceed with caution in exome and genome sequencing studies. Genome Med. 2016;8(1):14.

47. Chalouhi N, Ali MS, Jabbour PM, Tjoumakaris SI, Gonzalez LF, Rosenwasser $\mathrm{RH}$, et al. Biology of intracranial aneurysms: role of inflammation. J Cereb Blood Flow Metab. 2012;32(9):1659-76.

48. Dixon JR, Selvaraj S, Yue F, Kim A, Li Y, Shen Y, et al. Topological domains in mammalian genomes identified by analysis of chromatin interactions. Nature. 2012;485(7398):376-80.
49. Schmitt Anthony D, Hu M, Jung I, Xu Z, Qiu Y, Tan Catherine L, et al. A Compendium of Chromatin Contact Maps Reveals Spatially Active Regions in the Human Genome. Cell Rep. 2016;17(8):2042-59.

50. Sauerwald N, Singhal A, Kingsford C. Analysis of the structural variability of topologically associated domains as revealed by Hi-C. NAR Genom Bioinform. 2020;2(1):lqz008. https://doi.org/10.1093/nargab/lqz008.

51. Sauerwald N, Kingsford C. Quantifying the similarity of topological domains across normal and cancer human cell types. Bioinformatics. 2018:34(13):i475-83.

52. Igolkina AA, Zinkevich A, Karandasheva KO, Popov AA, Selifanova MV, Nikolaeva D, et al. H3K4me3, H3K9ac, H3K27ac, H3K27me3 and H3K9me3 Histone tags suggest distinct regulatory evolution of open and condensed chromatin landmarks. Cells. 2019:8(9):1034.

53. Hnisz D, Day DS, Young RA. Insulated neighborhoods: structural and functional units of mammalian gene control. Cell. 2016;167(5):1188-200.

54. Humphrey JD, Taylor CA. Intracranial and abdominal aortic aneurysms: similarities, differences, and need for a new class of computational models. Annu Rev Biomed Eng. 2008;10:221-46.

55. Jamous MA, Nagahiro S, Kitazato KT, Satoh K, Satomi J. Vascular corrosion casts mirroring early morphological changes that lead to the formation of saccular cerebral aneurysm: an experimental study in rats. J Neurosurg. 2005;102(3):532-5.

56. Nixon AM, Gunel M, Sumpio BE. The critical role of hemodynamics in the development of cerebral vascular disease. J Neurosurg. 2009;112(6):1240-53.

57. Meng H, Tutino VM, Xiang J, Siddiqui A. High WSS or low WSS? Complex interactions of hemodynamics with intracranial aneurysm initiation, growth, and rupture: toward a unifying hypothesis. Am J Neuroradiol. 2014;35(7):1254.

58. Gates LA, Shi J, Rohira AD, Feng Q, Zhu B, Bedford MT, et al. Acetylation on histone $\mathrm{H} 3$ lysine 9 mediates a switch from transcription initiation to elongation. J Biol Chem. 2017;292(35):14456-72.

59. Dunham I, Kundaje A, Aldred SF, Collins PJ, Davis CA, Doyle F, et al. An integrated encyclopedia of DNA elements in the human genome. Nature. 2012:489(7414):57-74.

60. Dallas LS, David JM, Omar E, Jean-Paul B, Evan L, Stephanie HC, et al. Endothelial dysfunction in cerebral aneurysms. Neurosurgical Focus FOC. 2019:47(1):E3.

61. Kroon M, Holzapfel GA. A theoretical model for fibroblast-controlled growth of saccular cerebral aneurysms. J Theor Biol. 2009;257(1):73-83.

62. Fowlkes JL, Enghild JJ, Suzuki K, Nagase H. Matrix metalloproteinases degrade insulin-like growth factor-binding protein-3 in dermal fibroblast cultures. J Biol Chem. 1994;269(41):25742-6.

63. Xiaoran Z, William JA, Philipp T, Andrew FD, Ramesh G. Role of matrix metalloproteinases in the pathogenesis of intracranial aneurysms. Neurosurgical Focus FOC. 2019;47(1):E4.

64. Sotoodehnejadnematalahi F, Burke B. Structure, function and regulation of versican: the most abundant type of proteoglycan in the extracellular matrix. Acta Med Iran. 2013;51(11):740-50.

65. Douville JM, Cheung DYC, Herbert KL, Moffatt T, Wigle JT. Mechanisms of MEOX1 and MEOX2 regulation of the cyclin dependent kinase inhibitors p21 and p16 in vascular endothelial cells. PLoS ONE. 2011;6(12):e29099-e.

66. Hosaka K, Rojas K, Fazal Hanain Z, Schneider Matheus B, Shores J, Federico $\checkmark$, et al. Monocyte chemotactic protein-1-interleukin-6-osteopontin pathway of intra-aneurysmal tissue healing. Stroke. 2017;48(4):1052-60.

67. Zheng S, Su A, Sun H, You C. The association between interleukin- 6 gene polymorphisms and intracranial aneurysms: a meta-analysis. Hum Immunol. 2013;74(12):1679-83.

68. Ahituv N. Exonic enhancers: proceed with caution in exome and genome sequencing studies. Genome Medicine. 2016;8(1):14.

\section{Publisher's Note}

Springer Nature remains neutral with regard to jurisdictional claims in published maps and institutional affiliations. 